\title{
Intracellular Alterations of the Creatine Kinase Isoforms in Brains of Schizophrenic Patients
}

\author{
Tatyana P. KlushniK, ${ }^{* 1}$ Alexander Ya. SpUnde, \\ AlEXANDER G. YAKOVLEV,' ZAZA A. KHUCHUA, ${ }^{2}$ \\ VALDUR A. SAKS, ${ }^{2}$ AND MARAT E. VARTANYAN ${ }^{1}$
}

'All-Union Research Center for Mental Health, Acad. Med. Sci., Zagorodnoe, Shosse, 2, Moscow, 113152, USSR, '2All-Union Research Center for Cardiology, Acad. Med. Sci., Moscow, USSR

Received April 1, 1991; Accepted July 22, 1991

\begin{abstract}
Postmortem brain tissues of schizophrenic patients were found to contain 5-10 times less water-soluble creatine kinase (BB CK) and 1.5-3 times less mitochondrial creatine kinase as compared to control. The major part of BB CK in schizophrenic brain tissues, contrary to control, was found to be insoluble in water (particulate form of $B B$ $\mathrm{CK}$ ) and could be extracted from brain tissue with strong denaturating agents. The particulate form of BB CK did not have any enzymatic activity but activity was found after the solubilization of this isoenzyme. The observed BB CK translocation into the particulate inactive form and the decrease of mitochondrial CK content to schizophrenic brains may reflect changes in the synthesis and the utilization of creatine phosphate.
\end{abstract}

Index Entries: Human brain; schizophrenia; creatine kinase; enzyme translocation.

*Author to whom all correspondence and reprint requests should be addressed. 


\section{INTRODUCTION}

Creatine kinase (CK, EC 2.7.3.2) is one of the most important enzymes in the energy metabolism of many cell types including those of brain (Saks et al., 1978; Wallimann et al., 1990). It maintains high local concentrations of ATP by a rapid rephosphorylation of ADP at the expense of phosphocreatine (Wallimann and Eppenberger, 1990).

Several isoforms of CK were described and characterized by tissuespecific expression. One of them, the brain isoenzyme (CK BB), is expressed in a wide variety of tissues with the highest level detected in the brain (Quest et al., 1990). The expression of CK BB is under the control of several regulatory factors including peptides, steroid hormones, and transforming factors (Kaye, 1986; Reiss, 1988).

The previous analysis of protein composition of water-soluble brain extracts by two-dimensional polyacrylamide gel (PAG) electrophoresis has revealed significant differences between the contents of $C K B B$ in the normal and schizophrenic human brains taken by autopsy (Klushnik et al., 1991).

The purpose of this work was to investigate in detail the reason for this difference by using a method of fractional extraction of creatine kinase from the brain.

\section{MATERIALS AND METHODS}

The diagnosis of schizophrenia was based on the clinical observations in accordance with the international disease classification (ICD 9).

Ten patients were taken for the analysis in each of the two following groups:

Group 1: Patients who had no evidence of mental disorders and who died from sudden heart attack. This group served as the normal control.

Group 2: Patients with different forms of schizophrenia who died from sudden heart attack or pneumonia. Table 1 summarizes the clinical features of these patients. The diagnosis of schizophrenia was specified by psychiatrists, who observed the patients during a long period of time in the All-Union Mental Health Research Center. The psychiatrists used the generally accepted classification of JCD-9 with the rubrification of schizophrenia under 295.4-acute schizophrenia attack and 295.3paranoid schizophrenia. Most of the patients who were observed in clinical conditions were treated with neuroleptic drugs (haloperidol, chlorpromazine, stelazine) in combination with antidepressive preparations (amithriptilline, melipromine) in medium therapeutical dosages.

Human brain tissues were obtained from autopsy and frozen at $-70^{\circ} \mathrm{C}$. Samples of frontal cortex (area 10 according to Brodman) from different patients were studied in each group. 
Table 1

Clinical Data Pertinent to Tissue Sources

\begin{tabular}{lcc}
\hline & Normal & Schizophrenia \\
\hline No. of patients & 10 & 9 \\
Sex & $7 \mathrm{M}+3 \mathrm{~F}$ & $3 \mathrm{M}+6 \mathrm{~F}$ \\
Age (y) & $56.7 \pm 4.0$ & $63.7 \pm 3.2$ \\
Diagnosis (ICD) & - & $295.4 ; 295.3$ \\
Hours, postmortem & $5.9 \pm 0.5$ & $7.4 \pm 0.7$ \\
\hline
\end{tabular}

Some 100-200 mg of frozen brain tissues were homogenized in $10 \mathrm{vol}$ of $100 \mathrm{mM}$ potassium phosphate buffer (KPB), $\mathrm{pH} 9$, containing $0.2 \mathrm{mM}$ EDTA, $5 \mathrm{mM}$ dithiothreitol, and $0.1 \%$ Nonidet P40 in Ultraturax homogenizer. The homogenate was centrifuged at $16000 \mathrm{~g}$ for $30 \mathrm{~min}$. The supernatant containing KPB-soluble proteins was analyzed for the enzyme activities and by electrophoresis.

The pellet was washed once with KPB, resuspended in initial vol of the same buffer containing $8 M$ urea (Urea-KPB) and centrifuged as described earlier. Supernatant obtained contained proteins solubilized in urea but not in KPB. Immediately after centrifugation $0.5-1 \mathrm{~mL}$ of the supernatant was applied on NAP-5 Column (Pharmacia, Sweden) to remove urea. After this procedure, a fraction of urea-soluble proteins was used in the enzyme activity and electrophoretic analysis.

For the quantitative measurement of a CK activity in KPB-soluble and urea-soluble fractions we used NAC-activated CPK test (KONE Corp., Finland).

Immunoelectroblotting was performed as described earlier (Towbin et al., 1979). Samples of KBP-soluble and urea-soluble proteins were subjected to SDS-electrophoresis (Laemmli, 1970) in 10\% PAG followed by the electrophoretic transfer to nitrocellulose membrane. Immunodetection of CK enzymes on nitrocellulose was performed with polyclonal antibodies against human brain CK BB developed in rabbit (Klushnik et al., 1991) and polyclonal antibody to human heart mitochondrial CK (Saks et al., 1987) using antirabbit IgG conjugated with peroxidase (Sigma).

Electrophoresis in the agarose gel was carried out as described earlier (Khuchua et al., 1989). Visualization of CK isoenzymes was performed by using a modified commercial method of fluorescence staining technique (CIBA Corning Diagnostics Corp., Palo Alto, CA, USA).

All results were analyzed by using Student's $t$-test. Mean values and standard deviation of parameters studied are given in Table 1 and Figs. 1 and 2, which also show the statistical significance of the difference observed. 


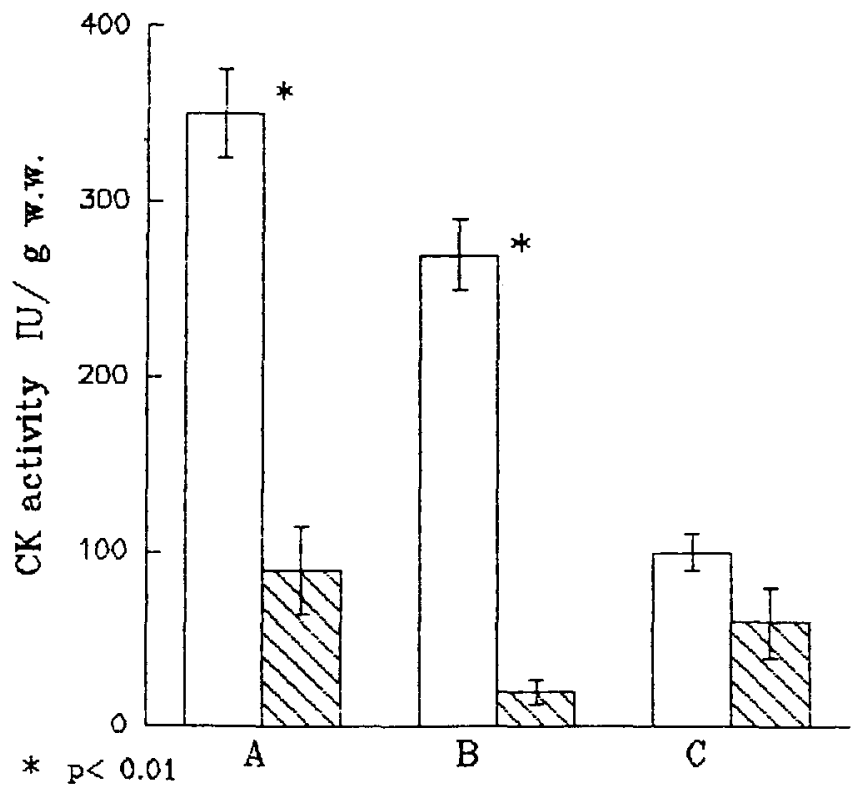

Fig. 1. KPB-extracted CK activities in normal $(\square)$ and schizophrenic $(\bowtie)$ brains. Total CK activity (A) was directly measured in tissue KPB extracts. The activities of $B B$ CK (B) and mitochondrial CK (C) were calculated on the basis of the total CK activity (A) and relative content of CK isoenzymes shown in Fig. 2.

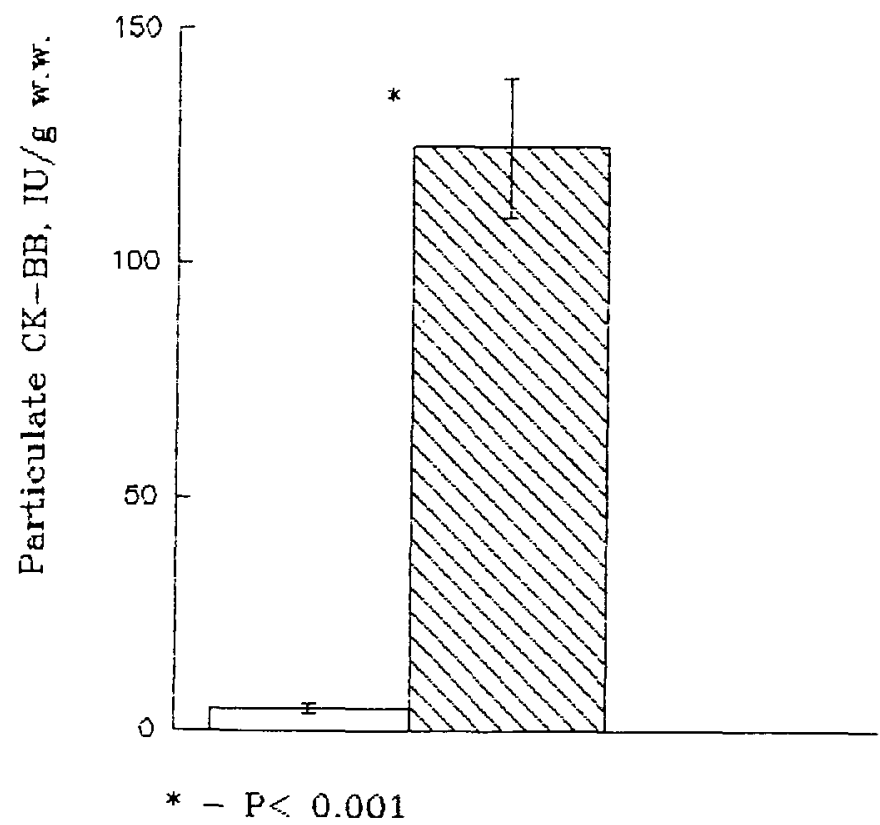

Fig. 2. The BB CK activity of particulate (urea-extractable) fraction from normal $(\square)$ and schizophrenic ( $₫)$ brains. The activity was calculated as the difference between CK BB extracted with $8 M$ urea and $\mathrm{KPB}$-soluble BB CK fractions activities. 


\section{RESULTS}

The results of immunoelectroblotting presented in Fig. 3 demonstrate that the content of BB CK is significantly decreased in the KPBextracts of schizophrenic brains. Estimation of enzyme activities in the extracts of frontal cortex of both normal and schizophrenic brains showed that the activity of CK is stable to autolysis and practically was not changed during 3-10 $\mathrm{h}$ of postmortem period. Total CK activity in the $\mathrm{KPB}$-extracts of normal brain was equal to $356 \pm 25 \mathrm{IU} / \mathrm{g}$ wet wt. In the KPB-extracts of schizophrenic brains, the activity of CK was significantly decreased and was equal to $83 \pm 23 \mathrm{IU} / \mathrm{g}$ wet wt. Inasmuch as it is known that brain may contain different CK isoenzymes (Wevers et al., 1981), it was of interest to estimate the CK isoenzyme spectrum of the total KPBextracts. The results of quantitative electrophoresis of KPB-extracts of the brain in agarose gels are shown in Fig. 4.

Two major isoforms of the $\mathrm{CK}$ were found in these extracts, i.e., BB and the mitochondrial $\mathrm{CK}$. Tissues taken from normal individuals contained $75-85 \%$ BB and 15-25\% of mitochondrial form, but in the KPBextracts of brain of schizophrenic patients this ratio was reversed (Fig. 4). On the basis of obtained ratio and total activity of CK in protein extracts we could calculate the values of activities for both CK forms (Fig. 1). The calculated CK activity values demonstrated that the activity of BB CK is decreased 5-10 times in the schizophrenic brains as compared to control. The activity of mitochondrial isoform was also decreased but to a lesser extent. These data are in good agreement with the results of immunoblotting shown in Fig. 3.

In order to understand if observed phenomenon is a result of the real decrease of CK isoenzymes content or a result of changes in the protein solubility, the brain tissues after the primary extraction with $100 \mathrm{mM} \mathrm{KPB}$ were repeatedly extracted with $8 M$ urea and/or mercaptoethanol. These extractions resulted in the additional release of $B B C K$. Such a release was not observed when KPB with different $\mathrm{pH}$ or containing $1-5 \%$ Triton $X-100$ were used. These results indicate that the total population of CK $\mathrm{BB}$ in the brain of schizophrenic patients is presented by both KPBsoluble and membrane-bound fractions. The latter one can be extracted only by using strong denaturating agents. The results of immunoblotting illustrating distribution of $\mathrm{BB} C \mathrm{CK}$ isoforms between phosphate-soluble and urea-soluble fractions are shown in Fig. 5.

The comparative analysis of KPB and urea extracts of brains revealed that the mitochondrial form of CK is completely extracted by $100 \mathrm{mM}$ phosphate solution, $\mathrm{pH}$ 9.0, inasmuch as application of antiserum showed the presence of trace amounts of this isoenzyme in urea-containing extracts in normal and in schizophrenic brains. Along with this, it is important to note that the amount of this CK isoenzyme is decreased 1.5- 


\section{$\begin{array}{llllllllll}\text { I } & 2 & 3 & 4 & 5 & 6 & 7 & 8 & 9 & \text { IO }\end{array}$}

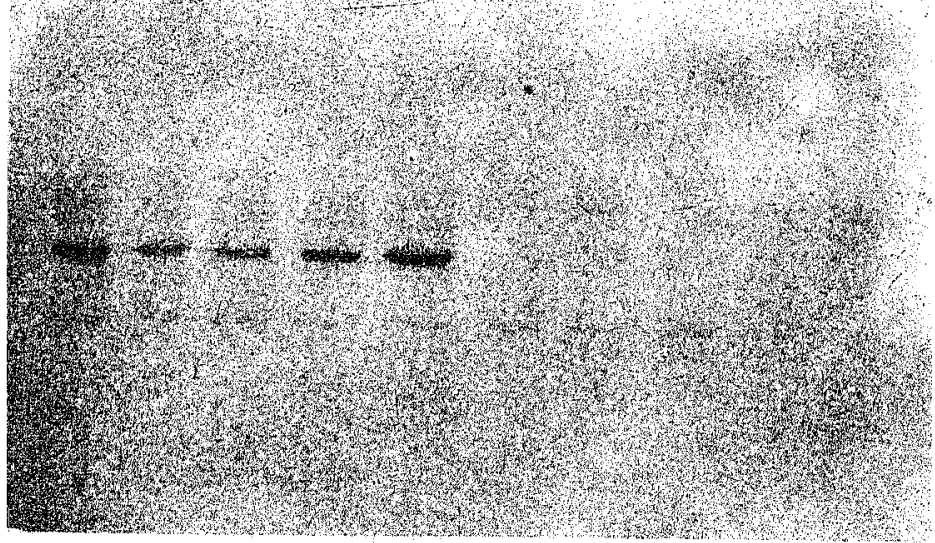

Fig. 3. The immunoblotting of BB CK from normal and schizophrenic brains extracted with potassium phosphate buffer. Numbers correspond to different patients. Proteins were extracted from normal (lines 1-5) and schizophrenic human brains (lines 6-10) with $100 \mathrm{mM}$ potassium phosphate buffer and after SDS-electrophoresis were transferred to nitrocellulose membrane. All lanes contain equal quantity of total protein $(20 \mu \mathrm{g})$. Immunodetection was carried out with polyclonal antibodies against human brain BB CK using antirabbit IgG conjugated with peroxidase (see Materials and Methods).
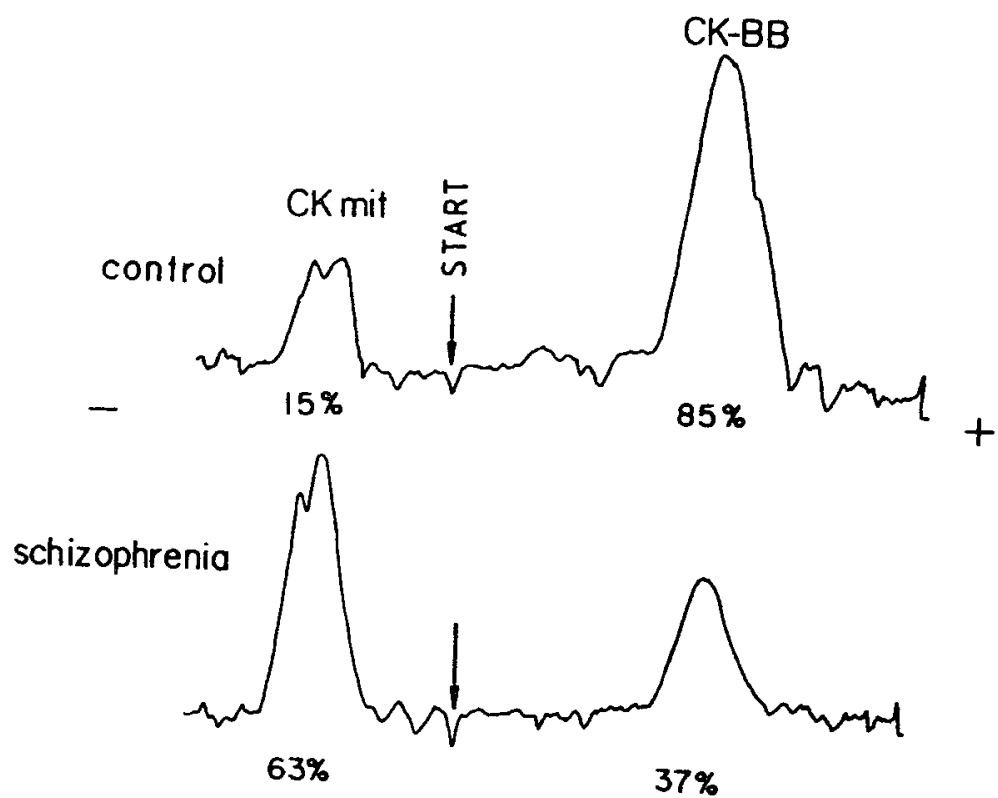

Fig. 4. Fluorescence profiles of CK isoenzymes from normal and schizophrenic brains after electrophoresis in agarose gel. Buffer: $60 \mathrm{mM}$ Tris- $\mathrm{HCl} / 10 \mathrm{mM}$ barbital/50 $\mathrm{mM}$ sodium barbital/1 $\mathrm{m} M$ EGTA/1 $\mathrm{m} M$ dithiothreitol/0.1\% Triton $\mathrm{X}-100(\mathrm{pH} 9.0) ; 100 \mathrm{~V}$ for $30 \mathrm{~min}$ at $4^{\circ} \mathrm{C}$. 


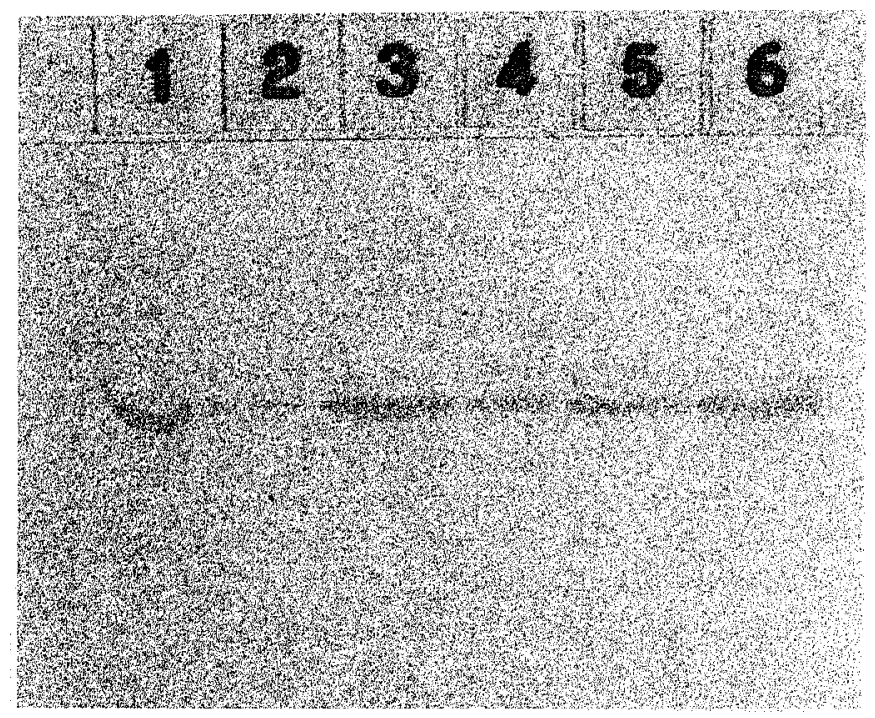

Fig. 5. The immunoblotting of CK BB from normal and schizophrenic brains extracted with $\mathrm{KPB}$ and with $8 M$ urea. Lines 1-3: Proteins extracted from the brain of a normal patient. 1-extraction with $\mathrm{KPB}, 2$ subsequent extraction with $8 M$ urea, 3 -direct extraction with $8 M$ urea (total fraction). Lines 4-6: Proteins extracted from the brain of a schizophrenic patient. 4 extraction with $\mathrm{KPB}, 5$-subsequent extraction with $8 M$ urea, 6-direct extraction with $8 M$ urea.

3 times in schizophrenic brains as compared to control. As shown in Fig. 2 , the major part of BB CK isoenzyme in normal brains was extracted by $100 \mathrm{mM}$ phosphate and only trace amount is additionally extracted by solution containing $8 M$ urea. In the case of schizophrenia the latter represents about $75 \%$ of the total amount of BB CK. The particulate (ureaextractable) fraction obtained after primary extraction of brain tissue with $\mathrm{KPB}$ solution did not show $\mathrm{CK}$ activity that reappeared only after solubilization of the enzyme with urea solution. Thus, the particulate BB CK seems to be enzymatically inactive.

Chlorpromazine and haloperidol used in regular accepted doses per $\mathrm{kg}$ body wt in rats for $30 \mathrm{~d}$ did not result in alteration of $\mathrm{CK}$ activity.

\section{DISCUSSION}

The present results show that BB CK in the brain of schizophrenic patients is modified or translocated from easily extractable active form into particulate inactive form. 
Two isoenzymes of CK: mitochondrial $\mathrm{CK}$ and $\mathrm{BB} C \mathrm{CK}$, compose a phosphocreatine pathway of energy transport in the brain cells that is similar to the analogous pathway in cardiac, skeletal muscle cells and other cell types (Saks et al., 1978; Wallimann and Eppenberger, 1990). The phosphocreatine pathway has been described in detail for cardiomyocytes because of easiness of quantitative determination of heart function. The idea of this "phosphagen" pathway was also formulated for nervous cells by A. Hodgkin in 1964 on the basis of experimental data showing a clear preference of $\mathrm{Na}^{+}, \mathrm{K}^{+}$-pump of giant axon for phosphoarginine (+ ADP) in comparison with ATP as an energy source (Hodgkin, 1964). In 1964 Klingenberg and his associates (see Jacobs et al., 1964) described the presence in the brain of two CK isoforms: mitochondrial and $\mathrm{BB}$. Mitochondrial $\mathrm{CK}$ is attached to the outer surface of the inner mitochondrial membrane and may acquire octameric form (Schlegel et al., 1990). These forms, however, do not differ in enzymic activity. It uses mitochondrial ATP for phosphocreatine synthesis, which is an energy carrier inside the cells (Jacobus, 1985). BB CK may be located in or connected to the surface membrane near by $\mathrm{Na}^{+}, \mathrm{K}^{+}$-ATPase to replenish the local pool of ATP at the expense of phosphocreatine synthesized in mitochondria (Saks et al., 1978).

Mitochondrial CK can be easily detached from the membrane with $100 \mathrm{mM} \mathrm{KPB}, \mathrm{pH} 8.0-9.0$ (Jacobus, 1985). We observed that about $90 \%$ of $\mathrm{BB} C \mathrm{CK}$ is extracted (detached from membrane) from normal brain under similar conditions. Such an easiness of BB CK extraction with low molarity saline buffers was also reported by other authors (Chandler, 1987; Miller and Wei, 1985).

In the brain of schizophrenic patients, BB CK is converted or translocated almost completely into particulate inactive form that may be solubilized and reactivated with strong denaturating agents. These changes concern specifically BB CK and are not related to mitochondrial CK. A decrease of the latter enzyme activity by a factor $1.5-3$ probably shows serious disturbances in the phosphocreatine synthesis, which is the first step in the energy transport. Conversion of the major part of BB CK into particulate inactivated form may reflect defects in the energy conversion at the level of cellular membranes. Apparently, obtained results testify to serious defects in the system of intracellular energy supply in the schizophrenic brain. Earlier, it has been shown that glucose utilization is decreased in this disease.

A cause of CK isoenzyme alteration is not known as yet. We did not find any correlation between the activity of $\mathrm{BB} \mathrm{CK}$ in KPB-soluble fraction and a clinical form of schizophrenia, the sex and the age of patients, or the postmortem interval. We believe that the examination of molecular and cellular mechanisms of the $\mathrm{BB} C \mathrm{CK}$ translocation deserve further attention and study. 


\section{SUIMMARY}

The content and the enzymatic activity of $\mathrm{CK}$ isoenzymes (BB CK and mitochondrial $(\mathrm{CK}$ ) were estimated in postmortem normal and schizophrenic brains. Water-soluble extracts of schizophrenic brain tissues contain 5-10 times less of BB CK and 1.5-3 times less of mitochondrial $\mathrm{CK}$ as compared to the control. The extraction of the particulate fraction of schizophrenic brain tissues with $8 M$ urea and 2-mercaptoethanol resulted in the additional release of $\mathrm{BB} C \mathrm{CK}$ but not of mitochondrial CK. The particulate form of BB CK does not have any enzymatic activity, which can be restored after solubilization and subsequent elimination of urea. The observed translocation of BB CK from easily extractable active form into particulate inactive form and the decrease of mitochondrial CK content in schizophrenic brian may reflect the changes in the synthesis and the utilization of creatine phosphate.

\section{REFERENCES}

Chandler W. L. (1987) Comparison of solutions for extracting CK, AK and LD from brain samples. Clin. Chem. 33, 2304-2306.

Hodgkin A. L. (1964) The Conduction of Nervous Impulse. Liverpool University Press. Liverpool, pp. 80-85.

Jacobs H., Heldt H. W., and Klingenberg M. (1964) High activity of creatine kinase in mitochondria from muscle and brain and evidence for a separate mitochondrial isoenzyme of creatine kinase. Biochem. Biophys. Res. Comm. 16, 516-521.

Jacobus W. E. (1985) Respiratory control and the integration of heart highenergy phosphate metabolism by mitochondrial creatine kinase. Ann. Rev. Physiol. 47, 707-725.

Kaye A. M. (1986) Hormonal regulation of creatine kinase BB. Adv. Exp. Med. Biol. 194, 83-101.

Khuchua Z. A., Ventura-Clapier R., Kuznetsov A. V., Grishin M. N., and Saks V. A. (1989) Alterations in the creatine kinase system in the myocardium of cardiomyopathic hamsters. Biochem. Biophys. Res. Comm. 165, 748-757.

Klushnik T. P., Spunde A. Ya., and Yakovlev A. G. (1991) Decreasing of creatinephosphokinase BB in water-soluble fraction of schizophrenic brains revealed by two-dimensional electrophoresis. Neurochimia, in press.

Laemmli U. K. (1970) Cleavage of structural proteins during the assembly of the head of bacteriophage T4. Nature 227, 680-685.

Miller J. and Wei R. (1985) Properties of creatine kinase from canine and human brain tissue. Clin. Biochem. 18, 14-19.

Quest A. F. G., Eppenberger H. M., and Wallimann T. (1990) Two different B-type creatine kinase subunits dimerize in a tissue specific manner. FEBS Lett. 262, 299-304. 
Reiss N. A. (1988) Ontogeny and estrogen responsiveness of creatine kinase and glycolytic enzymes in brain and uterus of rat. Neurosci. Lett. 84, 197-202.

Saks V. A., Rosenshtraukh L. V., Smirnov V. N., and Chazov E. I. (1978) Role of creatine phosphokinase in cellular function and metabolism. Can J. Physiol. Pharmacol. 56, 691-706.

Saks V. A., Khuchua Z. A., and Kuznetsov A. V. (1987) Specific inhibition of ATP-ADP translocase in cardiac mitoplasts by antibodies against mitochondrial creatine kinase. Biochim. Biophys. Acta 891, 138-144.

Schlegel J., Wyss M., Eppenberger H., and Wallimann T. (1990) Functional studies with the octameric and dimeric form of mitochondrial creatine kinase. J. Biol. Chem. 265, 9221-9227.

Towbin H., Staehelin T., and Gordon J. (1979) Electrophoretic transfer of proteins from polyacrylamide gels to nitrocellulose sheets: Procedure and some applications. Proc. Natl. Acad. USA 76, 4350-4354.

Wallimann T. and Eppenberger H. M. (1990) The subcellular compartmentation of creatine kinase isoenzymes as a precondition for a proposed phosphorylcreatine circuit, in Isoenzymes: Structure, Function and Use in Biology and Medicine (Wallimann, T., ed.), pp. 877-889, Wiley-Liss, New York.

Wevers R. A., Reutelingsperger C. P. M., Dam B., and Soons J. B. J. (1981) Mitochondrial creatine kinase (EC 2.7.3.2) in the brain. Clin. Chim. Acta 119, 209-223. 